\title{
Electric polarization as a nonquantized topological response and boundary Luttinger theorem
}

\author{
Xue-Yang Song $\left(0,{ }^{1,2}\right.$ Yin-Chen He $\odot,{ }^{2}$ Ashvin Vishwanath, ${ }^{1}$ and Chong Wang ${ }^{2}$ \\ ${ }^{1}$ Department of Physics, Harvard University, Cambridge, Massachusetts 02138, USA \\ ${ }^{2}$ Perimeter Institute for Theoretical Physics, Waterloo, Ontario N2L 2Y5, Canada
}

(Received 22 February 2021; accepted 5 March 2021; published 2 April 2021)

\begin{abstract}
We develop a nonperturbative approach to the bulk polarization of crystalline electric insulators in $d \geqslant 1$ dimensions. Formally, we define polarization via the response to background fluxes of both charge and lattice translation symmetries. In this approach, the bulk polarization is related to properties of magnetic monopoles under translation symmetries. Specifically, in $2 \mathrm{D}$, the monopole is a source of $2 \pi$ flux, and the polarization is determined by the crystal momentum of the $2 \pi$ flux. In $3 \mathrm{D}$, the polarization is determined by the projective representation of translation symmetries on Dirac monopoles. Our approach also leads to a concrete scheme to calculate polarization in $2 \mathrm{D}$, which in principle can be applied even to strongly interacting systems. For open boundary conditions, the bulk polarization leads to an altered boundary Luttinger theorem (constraining the Fermi surface of surface states) and also to modified Lieb-Schultz-Mattis theorems on the boundary, which we derive.
\end{abstract}

DOI: 10.1103/PhysRevResearch.3.023011

\section{INTRODUCTION}

The bulk electric polarization of an insulator is a concept of fundamental importance in condensed matter physics. Polarization as a bulk quantity in ferroelectricity, piezoelectricity (polarization induced by mechanical stress), etc. have been widely studied in traditional solid-state physics. An important theoretical discovery that related (the change in) polarization to a geometric Berry phase [1-10] reveals its profound connection to topology properties in quantum systems such as quantum Hall effects and topological insulators. The precise definition and interpretation of polarization, however, is a subtle issue (see, for example, Refs. [3,11]).

Intuitively, the polarization measures the density of electric dipole moments in the bulk. Polarization density in a $d$-dimensional crystalline system with volume $V$ and charge density $\rho(r)$ reads

$$
\widehat{P}=\frac{1}{V} \int d^{d} r \rho(r) \mathbf{r}
$$

However, a surface charge distribution will induce a nonvanishing change in polarization per volume due to the position operator in the definition. Powerful methods that avoid such an issue have been established to calculate the polarization, at least within the independent electron approximation of band theory $[1,2,9,10,12,13]$. Periodic boundary conditions were instead adopted and polarization was calculated using Wannier functions of the occupied bands. It is also demonstrated that for a generic insulating system with interactions,

Published by the American Physical Society under the terms of the Creative Commons Attribution 4.0 International license. Further distribution of this work must maintain attribution to the author(s) and the published article's title, journal citation, and DOI. the change in polarization during an adiabatic evolution is well-defined and given by the integrated bulk currents, which could be further expressed as a many-body Berry phase [14].

In this paper, we develop a more topological approach to define and to directly measure the bulk polarization in arbitrary spatial dimensions. Specifically, we use a topological term to define polarization (per unit cell volume) in any dimensions and explore its consequences for both the periodic and open boundary conditions. For the periodic boundary conditions, the polarization determines the properties of magnetic monopoles under translation symmetries (such as momenta). For the open boundary condition, the bulk polarization determines the degree of Luttinger theorem violation on the boundary and more generally is related to quantum anomalies of the boundary low-energy theory. Our approach applies to short-range entangled systems of interacting fermions and bosons (or spins) as long as there are lattice translation symmetries and a conserved $U(1)$ charge with a charge gap (i.e., an insulator).

The paper is organized as follows. In Sec. II, we motivate a $1 \mathrm{D}$ expression of the topological term for polarization and discuss its various implications and issues for the generalization to higher dimensions. Section III introduces the notion of translation gauge fields and the topological term for polarization as a central result of this work. Section IV explores the connections of translation properties of magnetic monopoles and polarization density in 2D and 3D, implicated by the topological term in Sec. III. Section V discusses open systems where bulk polarization modifies the boundary Luttinger theorem. The relation of surface charge distribution to bulk polarization is further clarified through this discussion. Lieb-Schultz-Mattis (LSM)-type constraints descending from our topological term on topologically ordered systems and defect scenarios are also discussed. Section VI summarizes the results and provides physical arguments underlying the 
entire paper. The Appendices contain details on calculation recipes, subtleties on polarization, derivation and discussion of anomalies, and numerical results.

Before discussing the main results, we note that there is a feature in the definition of polarization when the unit cell has a nontrivial geometric structure. The polarization $\mathbf{P}$ consists of two parts: a classical electric dipole moment within each unit cell and an extra part that measures inter-unit-cell entanglement $[15,16]$ - the latter is denoted as $\tilde{\mathbf{P}}$ in Ref. [11]. Both $\mathbf{P}$ and $\tilde{\mathbf{P}}$ have been discussed in the literature depending on context. We review these notions briefly in Appendix A. Our results below can be applied to both the full $\mathbf{P}$ and to $\tilde{\mathbf{P}}$ as long as we adopt the appropriate calculation scheme as described below.

\section{POLARIZATION IN 1D}

Polarization has been $[14,17,18]$ formulated in $1 \mathrm{D}$ systems of size $L$ at integer fillings in terms of the expectation value of the large gauge transformation operator on the ground state (GS), i.e.,

$$
2 \pi P=\operatorname{Im} \ln \left\langle\mathrm{GS}\left|e^{i 2 \pi \widehat{P}}\right| \mathrm{GS}\right\rangle,
$$

where $\widehat{P}$ is the dipole moment density in Eq. (1) and the operator induces a gauge transform on the electron operator $c_{r} \rightarrow c_{r} e^{i 2 \pi r / L}$. Watanabe and Oshikawa [11] showed its equivalence to the Berry phase for a flux-piercing process of a $1 \mathrm{D}$ ring where flux $\theta$ adiabatically increases from 0 to $2 \pi$ (under an appropriate gauge choice of the GS). The electromagnetic field $A_{x}(r)=\theta / L$ increases as the flux pierces the system. In the $1+1 \mathrm{D}$ action, the $2 \pi P$ phase accumulation associated with the time-dependent $A_{x}(t)$ can be naturally written as $P \int d x d t \partial_{t} A_{x}$, where $\int d x A_{x}(t)$ increases from 0 to $2 \pi$. Hence, the polarization can be considered as an electromagnetic response defined by a topological $\Theta$ term in the low energy (IR) (setting $\hbar=c=e=1$ ),

$$
S_{P}^{1 \mathrm{D}}=P \int d x d t\left(\partial_{t} A_{x}-\partial_{x} A_{t}\right),
$$

where we added another term $\partial_{x} A_{t}$ to keep the gauge invariance. The flux-piercing process induces a change of $2 \pi$ in $\int d x A_{x}$ and hence a phase of $2 \pi P$ in the action. This term can also be motivated by the fact that a dipole moment $\mathbf{d}$ couples to electric field as $-\mathbf{d} \cdot \mathbf{E}$. Since $\int d A=\int d x d t\left(\partial_{t} A_{x}-\partial_{x} A_{t}\right)$ is always an integer (the first Chern number) multiple of $2 \pi$ on a closed two-manifold, $P$ is defined mod 1 . The periodicity of $P$ can be understood on the lattice by noticing that shifting an integer charge by one lattice unit (we set to be $a=1$ ) in every unit cell is equivalent to a relabeling of lattice coordinates and should not have any physical effect.

The polarization, defined via Eq. (3), has several consequences. First, for periodic systems, as discussed above, an adiabatic flux-threading process where $\int d x A_{x}$ changes by $2 \pi$ leads to a Berry phase $\Phi=2 \pi P$ from the space-time path integral of Eq. (3). This Berry phase is in principle a measurable quantity and is sometimes used as the definition of polarization in 1D [14,17]. For open boundary conditions, Eq. (3) becomes a boundary term $\pm P \int d t A_{t}$, which represents a fractional charge $q= \pm P(\bmod 1)$ at each boundary-the mod- 1 condition comes from the fact that one can always de- posit an integer charge on the boundary without affecting the bulk. This is consistent with the intuitive connection between polarization and dipole moments.

We note that for the Berry phase to be well-defined, the GS is required to return to itself up to a phase after an adiabatic flux threading, i.e., the GS space should be nondegenerate. As a counterexample, for fractional filling cases, according to LSM theorem, a gapped GS must break translation invariance. For a rational filling $p / q$ (irreducible fraction), it could be remedied by threading $2 \pi q$ flux and measure the polarization as the Berry phase divided by $q$, modulo $1 / q$ [18]. Raising this to higher dimensions poses some challenges. A simple generalization of the electromagnetic (EM) response term Eq. (3) to higher dimensions does not produce a topological term. One can consider it as a Berry phase term and measure the polarization through the Berry phase of a flux-threading process (say, in the $x$ direction) similar to that in 1D. The Berry phase, however, is given by

$$
\Phi_{x}=\frac{V}{L_{x}} 2 \pi P_{x} \quad(\bmod 2 \pi)
$$

where $V=L_{x} L_{y} \ldots$ is the system volume and $L_{x}$ is the length in the $x$ direction. If we assume lattice translation symmetries (which we do for the rest of the paper), the intensive quantity P can be extracted from the $L$ dependence of $\Phi$ (but simply dividing by $V / L_{x}$ will not work since the phase is defined mod $2 \pi)$. However, it raises the conceptual question whether $\mathbf{P}$ itself bears any physical meaning. For example, for a $2 \mathrm{D}$ crystalline insulator with $L_{y}=2 N(N \rightarrow \infty$ in thermodynamic limit) and a polarization density $P_{x}=1 / 2$, the Berry phase from Eq. (4) is always trivial. Is there a formula for the polarization in this case? One can always define polarization by starting with a reference state with a known polarization (for example, where polarization is constrained by symmetries) and connect it to the Hamiltonian of interest by an adiabatic path, and integrating the currents obtained while connecting the initial and final states. However, this algorithm requires defining such an adiabatic path and is conceptually different from a direct measure of polarization that we seek.

A similar issue appears with open boundary conditions: The density of dipole moment, which is the classical definition of polarization, is given by the surface charge density. Unlike the $1 \mathrm{D}$ case where the boundary charge is robustly determined mod 1 , the surface charge density in higher dimensions can be continuously tuned by boundary perturbations (for example, a boundary chemical potential). It then appears that the boundary does not necessarily reflect the bulk polarization. An exception was observed in Ref. [13]: When the boundary is gapped and nondegenerate, the boundary charge density faithfully represents the bulk polarization mod 1 . As we shall see, a topological approach is needed because polarization cannot be measured by local probes - something global, such as symmetry fluxes or physical boundaries, has to be introduced. This justifies the use of the term topological response, even though the response itself (the polarization) is in general not quantized and hence its value will change in response to symmetric perturbations. The familiar electromagnetic polarizability (the $\Theta$ term) in 3D also falls into this category when the time-reversal and mirror symmetry (or, more generally, 
symmetries that invert an odd number of space-time coordinates) are absent.

\section{POLARIZATION FROM TOPOLOGICAL TERMS}

Interesting IR physics can often be probed by the response to background gauge fields. In the study of polarization density in $d>1$, the relevant symmetries include charge conservation and lattice translation symmetries, so we shall consider coupling the system to gauge fields associated with these symmetries. For charge conservation, the gauge field is simply the electromagnetic field $A_{\mu}$. For each translation symmetry $\mathbb{Z}$, say in the $i$ th direction, we introduce a $\mathbb{Z}$-gauge field $x_{i}$. This translation gauge field [19] is less familiar, so we review it below. The gauge field $x_{i}$ is locally flat $\left(d x_{i}=0\right)$ so only its Wilson loops $\int_{C_{1}} x_{i} \in \mathbb{Z}$ over loops (or one-cycles, $C_{1}$ ) in space-time is meaningful-formally, this means that $x_{i} \in H^{1}(M, \mathbb{Z})$ where $M$ is the space-time manifold. Furthermore, just like the Wilson loops in other gauge theories, the integer $\int_{C_{1}} x_{i}$ measures the number of $\widehat{x}_{i}$ translations one has to go through to travel across $C_{1}$. To be more concrete, consider a path integral description, with dynamical degrees of freedom $\psi$ (bosonic or fermionic) defined in continuous time $t \in[0, T)$ and on discrete lattice sites $s$ in space,

$e^{-i S_{\mathrm{eff}}\left[A, x_{i}\right]}=\int D[\psi(s, t)] \exp \left(-i \sum_{s} \int d t \mathcal{L}_{s}[\psi, A]\right)$,

where we have used locality and translation symmetries to write the Lagrangian as a sum of local terms of identical form, $\mathcal{L}_{s}[\psi, A]$, which involves only fields near site $s$. We take periodic boundary conditions in space and time (so $M$ is a torus). The translation gauge fields enter the partition function by specifying exactly how the periodic boundary conditions are taken:

$$
\begin{aligned}
& \psi(s, t)=\psi\left(s+\widehat{x}_{j} \int_{i} x_{j}, t\right) \\
& \psi(s, t)=\psi\left(s+\widehat{x}_{j} \int_{t} x_{j}, t+T\right) .
\end{aligned}
$$

We now explain these equations in more detail. The Wilson loop of $x_{i}$ in the $\widehat{x}_{i}$ direction gives the lattice size $\int_{i} x_{i}=L_{i}$. For $j \neq i$, the number $\int_{i} x_{j}$ measures how much the slice of the lattice at $x_{i}=L_{i}$ is displaced along the $\widehat{x}_{j}$ direction before it is identified with the slice at $x_{i}=0$. Similarly, the time component $\int_{t} x_{i}$ measures the displacement of the entire lattice at $t=T$ before being identified with $t=0$. In other words, while the longitudinal parts of the translation gauge fields measure the lattice size, the transverse parts measure the quantized shear strains of the lattice in both space and time. We can also consider a $(d-2)$-dimensional defect in space, around which $\int x_{i}=n \neq 0$ : this is simply a lattice dislocation with Burgers vector $\mathbf{B}=n \widehat{x}_{i}$.

The translation gauge field $x_{i}$ is closely related to the concept of tetrad in the theory of elasticity [20], which has been used to characterize three-dimensional integer quantum Hall effect recently [21-23] and torsions in Weyl semimetals [24]. Consider embedding the lattice into a continuous space, so each site $s$ can be assigned a continuous coordinate $\mathbf{u}_{s}$. We can treat $\mathbf{u}$ as a field, then the tetrad $\nabla u_{i}$ will have all the properties of $x_{i}$ discussed above and can be used as a representation (a gauge choice) of $x_{i}$. The gauge-invariant properties of $x_{i}$ such as the Wilson lines, however, do not depend on how the lattice is embedded into a continuous space. In this sense, the $x_{i}$ gauge field measures the topological part of the elasticity response. Another straightforward way is to consider the strain tensor $\partial_{i} d_{j}(\mathbf{r})$ in elasticity theory [25,26], where the displacement fields $\mathbf{d}(\mathbf{r})$ for site $\mathbf{r}$ are defined modulo the lattice spacing, i.e., a relabeling of sites by an integer vector $\mathbf{N}(\mathbf{r})$ does not make a physical difference. This invariance calls for the gauge field $x_{i}$,

$$
\begin{aligned}
d_{i}(\mathbf{r}) & \rightarrow d_{i}(\mathbf{r})+N_{i}(\mathbf{r}),\left(N_{i} \in \mathbb{Z}\right), \\
\nabla d_{i} & \rightarrow \nabla d_{i}+x_{i},
\end{aligned}
$$

where $x_{i}=\nabla N_{i}$ is defined on a discrete lattice and can be viewed as the translation gauge field. Now recall that the electric polarization in 1D can be defined through the topological term $P \int d A$ [Eq. (3)]. The natural generalization to higher $(d+1)$ dimensions is the following term:

$S_{\text {polar }}=\sum_{i}(-1)^{i+1} P_{i} \int x_{1} \wedge \ldots x_{i-1} \wedge d A \wedge x_{i+1} \ldots \wedge x_{d}$.

Here $\wedge$ should really mean cup product $\smile$ for discrete cohomology instead of the usual wedge product, but the distinction does not matter for our purpose.

We now give some justifications for Eq. (8) as a definition of bulk polarization. First, it is the only topological term involving $d A$ and $x_{i}$ that is first order in the field strength $d A$, as we expect for the polarization. Each component of polarization $P_{i}$ is defined $\bmod 1$ since the integral always gives integral multiples of $2 \pi$ on closed manifolds (the term is therefore a topological $\Theta$ term), and is in agreement with the intuition that shifting integer charges by one lattice unit does not have a physical effect. We note that for a system with spin degeneracy, in principle, two EM fields $A_{\uparrow, \downarrow}$ could be used to couple to the phases of spin-up, -down electron operators, respectively. Consequently, two topological terms with coefficients $P_{\uparrow, \downarrow}$ are present and each of the two polarization quantities is defined mod 1 . Total polarization density $P=P_{\uparrow}+P_{\downarrow}$ is defined $\bmod 2$. When evaluated for a uniform electric field $\mathbf{E}$ on a perfect lattice (free of dislocation and shear strain) of size $L_{1} \times L_{2} \ldots$, this term becomes

$$
S_{\text {polar }}\left(L_{i}\right)=\left(V / L_{i}\right) \sum_{i} P_{i} \int d t d x_{i} E_{i},
$$

which agrees with the expectation that the total polarization is $\left(V / L_{i}\right) P_{i}$ when the system is viewed as $1 \mathrm{D}$ in $\widehat{x}_{i}$ direction. In addition, if $\mathbf{P}$ has a time-dependence $\mathbf{P}(t)$, then by taking derivative with respect to $\mathbf{A}$ from the above action we obtain the charge current $\mathbf{j}=\partial \mathbf{P} / \partial t$, which agrees with physical expectations and is sometimes used as a practical way to define polarization. If $\mathbf{P}$ is spatially dependent, say varying in the $\widehat{x}_{i}$ direction, taking derivative with respect to $A_{t}$ on the term $(-1)^{i+1} \int P_{i} x_{1} \wedge \cdots x_{i-1} \wedge d A \wedge x_{i+1} \cdots \wedge x_{d}$ gives $-\rho=\partial_{x i} P_{i}$, agreeing with the relation $-\rho=\nabla \cdot \mathbf{P}$ [27] This integrating by part may leave a boundary term which accounts for the change of surface bound charge $\sigma_{i}$ due to spatial variation of polarization through $\sigma_{i}=\mathbf{P} \cdot \widehat{n}$, where $\widehat{n}$ is the normal vector of the surface. We emphasize that while 
the special or temporal variations of $\mathbf{P}$ results in locally measurable quantities like $\rho$ or $\mathbf{j}$, the more subtle constant piece of $\mathbf{P}$ comes with an intrinsically topological nature and needs to be defined via the topological term Eq. (8).

We note that the translation gauge field can also be used for magnetic translations. Suppose we have a lattice system in which each unit cell traps a U(1) magnetic flux $\phi$ in the $x y$ plane, then in our formulation the Dirac quantization condition for the $\mathrm{U}(1)$ gauge field is now changed to

$$
\int_{C_{2}}(d A-\phi x \wedge y)=0(\bmod 2 \pi),
$$

where $C_{2}$ represents arbitrary two-cycles in space-time. Most of our discussions in this paper will be equally applicable for magnetic translations as long as the above modified Dirac quantization condition is imposed.

\section{POLARIZATION AND MONOPOLES}

We now examine the consequences of the polarization as defined through Eq. (8) on a closed manifold (like periodic boundary conditions). Motivated by the $1 \mathrm{D}$ case, it is useful to consider instantons of the $A$ field. In 1D, the instanton is the familiar adiabatic flux-threading, a smooth configuration in space-time. In higher dimensions, the instantons become operators supported on $(d-2)$-dimensional submanifolds in space, with $\int d A=2 \pi$ on the two complementary spatial dimensions. For $d=2$, this is simply a unit flux insertion in space, and for $d=3$ it corresponds to a unit flux tube in space whose open ends become Dirac monopoles. On the $(d-2)+1$ manifold of the instanton, the topological terms reduces to the following Dijkgraaf-Witten [28] type:

$$
S_{\text {in }}=\sum_{i}(-1)^{i+1} 2 \pi P_{i} \int x_{1} \wedge \ldots x_{i-1} \wedge x_{i+1} \ldots \wedge x_{d} .
$$

Let us look at some physically relevant examples. At $d=2$, we obtain

$$
S_{\mathrm{in}, 2 \mathrm{D}}=2 \pi \int d t\left(P_{1} x_{2}^{(t)}-P_{2} x_{1}^{(t)}\right),
$$

which means that the $2 \mathrm{D}$ monopole-a point operator in space-carries charge of $2 \pi\left(-P_{2}, P_{1}\right)$ under translation symmetries in $\widehat{x}_{1}$ and $\widehat{x}_{2}$, respectively. But charge under translation symmetry is simply the crystal momentum. We then conclude that in 2D the monopole carries lattice momentum

$$
\mathbf{k}_{\mathcal{M}}=2 \pi\left(-P_{2}, P_{1}\right)=2 \pi \widehat{z} \times \mathbf{P} .
$$

It may be helpful to have some simple semiclassical picture here. Consider a $2 \pi$-flux quanta spread uniformly over a region much larger than the lattice unit. We can then consider the momentum of such a monopole $\mathbf{k}_{\mathcal{M}}$, i.e., the Berry phase from moving the unit flux configuration by one lattice unit. Equivalently, we can consider the many-body momentum of the fermions $\mathbf{k}_{e}$ under the flux configuration, which would be the inverse of the monopole momentum. Now imagine a semiclassical continuum system - a nonuniform electric field is induced during the turning on of the magnetic flux, which then induces a momentum on a small electric dipole moment $\mathbf{d}$ according to $\delta \mathbf{k} \sim \int d t(\mathbf{d} \cdot \nabla) \mathbf{E} \sim-\int d t \mathbf{d} \times(\nabla \times \mathbf{E}) \sim$

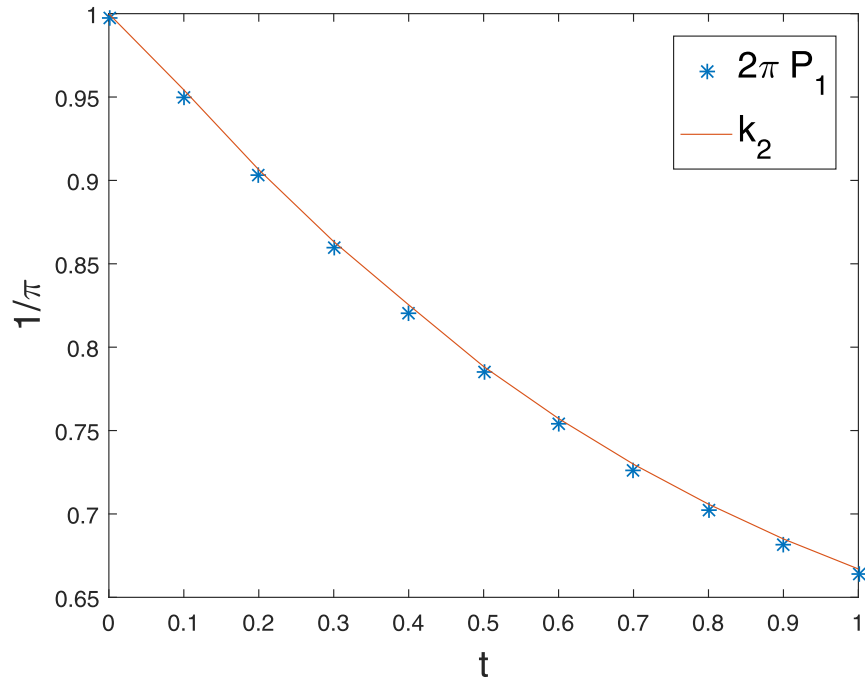

FIG. 1. The polarization $2 \pi P_{1}$ calculated from band theory and monopole momentum $k_{2}$ along the orthogonal direction always agree as one tunes a parameter $t$. Details can be found in Appendix B.

$\mathbf{d} \times \mathbf{B}$. Since the dipole density is given by $\mathbf{P}$, we have

$$
\mathbf{k}_{e}=-\mathbf{k}_{\mathcal{M}}=\int d^{2} r \mathbf{P} \times \mathbf{B}=2 \pi \mathbf{P} \times \widehat{z},
$$

which is what we obtained from the topological term.

We can use the monopole momentum as a practical way to calculate electric polarization in $2 \mathrm{D}$. We outline the calculation scheme here and discuss more details in Appendix B. Consider a 2D crystalline insulator on a torus, and smoothly spread a total magnetic flux of $2 \pi$ on the lattice-say $2 \pi / L_{x} L_{y}$ flux per plaquette. The total momentum of the many-electron system $\mathbf{k}_{e}$ can be measured from the GS wave function, as Berry phase factors associated with lattice translations, and from Eq. (14) we have $\mathbf{k}_{e}=2 \pi \mathbf{P} \times \widehat{z}$. The virtue of this calculational scheme is that it is well defined (although possibly complicated) even for strongly correlated systems, where band theory techniques cannot be used.

Another consequence of the connection between polarization and monopole momentum is that we can now define polarization in 2D even in the presence of gapless Dirac fermions. The only subtlety is that with a unit flux in space, each Dirac cone contributes a zero-energy mode, leading to multiple degenerate GSs depending on which zero modes are occupied. For each of the degenerate monopoles, we can nevertheless define its lattice momentum and interpret it as a bulk polarization, which then also depends on the zero-mode fillings. In Fig. 1, we report a numerical calculation of the polarization using the monopole momentum for a lattice system of gapless Dirac fermions with a specific choice of zero-mode filling. The same polarization can also be calculated using the standard method from band theory which we also report in Fig. 1 . The two results clearly agree as we vary a parameter $t$ in the Hamiltonian. More details of the calculational recipe and the lattice model can be found in Appendix B. In fact, there is a long history of numerically calculating monopole momenta for lattice Dirac fermions, motivated by the study of monopole operators in Dirac spin liquids [29-32]. We showed 

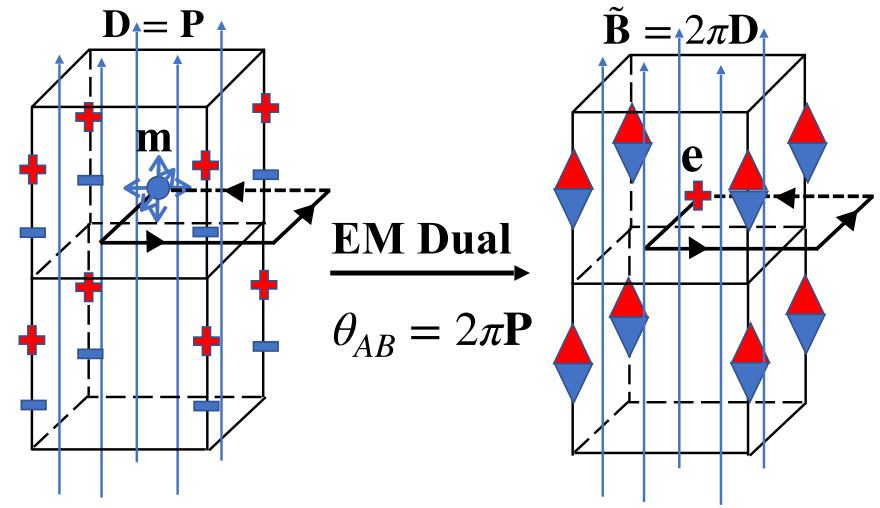

FIG. 2. The semiclassical picture relates projective representation of monopoles to polarization by electromagnetic duality that exchanges magnetic monopole and electric charge. The electric displacement field (left) $\mathbf{D}=\mathbf{P}$ is mapped to magnetic field (right) $\tilde{\mathbf{B}}=2 \pi \mathbf{D}$ and the monopole to an electric charge. The AharonomBohm (AB) phase $\theta_{\mathrm{AB}}$ seen by the electric charge is proportional to magnetic field (right), hence the monopole Berry phase proportional to displacement field (left).

that what this calculation really produces is the polarization of the underlying Dirac fermions.

For $d=3$, the term in Eq. (11) becomes a 2D integral,

$$
S_{i n, 3 \mathrm{D}}=\pi \epsilon^{i j k} \int P_{i} x_{j} \wedge x_{k},
$$

which describes a $2 \pi$ flux loop decorated with a $1 \mathrm{D}$ topological phase enriched by translation symmetries. This leads to nontrivial boundary modes when the flux loop has open ends, which are nothing but Dirac monopoles. The boundary mode is characterized by a projective representation [33-35] of translation symmetries, namely, translations in different directions commute up to a phase when acting on a Dirac monopole:

$$
T_{j}^{-1} T_{i}^{-1} T_{j} T_{i}=\exp \left(i 2 \pi \epsilon^{i j k} P_{k}\right) .
$$

This also has a simple semiclassical picture shown in Fig. 2. Consider a 3D continuum system with polarization density P. The polarization leads to an electric displacement field $\mathbf{D}=\mathbf{P}$. A magnetic monopole sees the $\mathbf{D}$ field as an effective dual magnetic field $\tilde{\mathbf{B}}=2 \pi \mathbf{D}=2 \pi \mathbf{P}$. A particle moving in an effective magnetic field realizes translation symmetries projectively, namely, different translation operations commute up to a phase factor according to Eq. (16).

Similar to the 2D case, the relation Eq. (16) is relevant for $U(1)$ quantum spin liquids in three dimensions, described at low energy by an emergent Maxwell U(1) gauge theory that is potentially realized, for example, in quantum spin ice materials [36]. Our results indicate that the monopoles in a $U(1)$ spin liquid will carry projective translation quantum numbers if the emergent electric charges form an insulator with nontrivial polarization density.

It was known from earlier approaches that polarization is related to other topological quantities including Hall conductance and magnetopolarizability (the axion angle). In Appendix (D2), we show that these connections can be easily understood using the polarization-monopole connection.
TABLE I. Polarization density $\mathbf{P}$ is related to the properties of the monopoles in dimensions $d=1,2,3$.

\begin{tabular}{lcc}
\hline \hline & Monopole property & Polarization \\
\hline 1D & Berry phase & $\Phi=2 \pi P$ \\
2D & Momentum & $\mathbf{k}_{\mathcal{M}}=2 \pi \widehat{z} \times \mathbf{P}$ \\
3D & Projective momentum & $T_{j}^{-1} T_{i}^{-1} T_{j} T_{i}=\exp \left(i 2 \pi \epsilon^{i j k} P_{k}\right)$ \\
\hline \hline
\end{tabular}

We summarize the connection between bulk polarization and monopole (instanton) properties in $d=1,2,3$ in Table I.

\section{BOUNDARY LUTTINGER THEOREM AND ANOMALY}

We now explore the consequences of bulk polarization for open boundaries. Consider a boundary at $x_{1}=0$ separating the vacuum at $x_{1}>0$ and the polarized bulk at $x_{1}<0$, which preserves all translation symmetries except the one along $\widehat{x}_{1}$. The $\Theta$-term in Eq. (8) becomes a boundary term:

$$
S_{\partial}=-P_{1} \int_{\partial} A \wedge x_{2} \ldots \wedge x_{d} .
$$

The meaning of this term can be seen by taking functional derivative with $A_{0}$ : It simply means a (fractional) charge density of $\rho_{\partial}=P_{1}$ on the boundary. If the boundary has trivial dynamics in the IR, namely, with a unique gapped GS, then Eq. (17) is the only nontrivial term in the IR description of the boundary. This is the well-known statement that for a trivially insulating boundary, the charge density is given by the bulk polarization density mod 1 [13].

In general, depending on details at the boundary, the boundary can also host nontrivial low-energy degrees of freedom. Let us first consider the simplest scenario: a Fermi liquid metal on the boundary. In this case, the boundary charge density $\rho$ is obviously not fixed by bulk polarization $P$ (in a direction perpendicular to the boundary) since it can be continuously tuned by perturbations that live only on the boundary. But we expect the Fermi surface volume $V_{\mathrm{F}}$ to be tuned simultaneously with the charge density following $\Delta V_{\mathrm{F}} /(2 \pi)^{d-1}=\Delta \rho$ from Luttinger theorem. We therefore expect

$$
\rho=\frac{V_{\mathrm{F}}}{(2 \pi)^{d-1}}+\mathbf{P} \cdot \widehat{\mathbf{n}}(\bmod 1),
$$

where $\widehat{\mathbf{n}}$ is the normal vector of the boundary. From this relation, the polarization density $P$ can be viewed as a source of Luttinger theorem violation on the boundary. Alternatively, we can view $V_{\mathrm{F}}$ as a quantum correction to the classical expectation of $P=\rho$. The boundary Luttinger theorem Eq. (18) can be understood using an anomaly-matching argument. It is useful to first phrase the usual Luttinger theorem in terms of anomaly matching, following ideas similar to those in Ref. [37]. Consider a theory in $(d-1)$ space dimensions of low-energy fermions near a Fermi surface, where fermion modes far away from the Fermi surface have been integrated out already. We couple the background gauge fields $A$ and $x_{i}$ minimally to these fermions and denote the action as $S_{\mathrm{FS}}\left[\psi, A, x_{i}\right]$. It is known that under a large gauge transform, in which the real space Wilson loop along the $\widehat{x}_{i}$ direction $\int_{C_{i}} A$ changes by $2 \pi$, the total crystal momentum of these low 
energy fermions changes by [37]

$$
\Delta \mathbf{K}=2 \pi \frac{V_{\mathrm{F}}}{(2 \pi)^{d-1}} L_{1} \ldots L_{i-1} \widehat{x}_{i} L_{i+1} \ldots L_{d-1} .
$$

This means that the theory $S_{\mathrm{FS}}\left[\psi, A, x_{i}\right]$ is not invariant under large gauge transforms. Instead, under a gauge transform $A \rightarrow A+d \alpha$, the low-energy theory near the Fermi surface transforms as

$$
S_{\mathrm{FS}}\left[\psi, A, x_{i}\right] \rightarrow S_{\mathrm{FS}}\left[\psi, A, x_{i}\right]+\frac{V_{F}}{(2 \pi)^{d-1}} \int d \alpha \wedge \prod_{i} x_{i} .
$$

To see Eq. (19) from Eq. (20), simply recall that $\int_{C_{i}} x_{i}=L_{i}$ and that the total momentum along $\widehat{x}_{i}$ is the coefficient of $\int d t x_{i}$. Equation (20) is also related to the familiar chiral anomaly in $(1+1)$ dimension, which we briefly explain in Appendix D Now, for a purely $(d-1)$-dimensional system that is not the boundary of another space, we should add a background term

$$
S_{\text {full }}=S_{\mathrm{FS}}\left[\psi, A, x_{i}\right]-\frac{V_{\mathrm{F}}}{(2 \pi)^{d-1}} \int A \wedge \prod_{i} x_{i},
$$

so the full theory is gauge invariant. The meaning of the counter term, as we discussed under Eq. (17), is simply a charge density of $\rho=V_{\mathrm{F}} /(2 \pi)^{d-1}$ — this is nothing but the familiar Luttinger theorem!

It is now straightforward to extend to the case of Eq. (18). Consider a Fermi liquid on the $(d-1)$ dimensional boundary of a $d$ dimensional bulk, with Fermi volume $V_{\mathrm{F}}$, and boundary charge density $\rho$. The surface theory reads

$$
\begin{aligned}
S_{\text {full }}= & S_{\mathrm{FS}}\left[\psi, A, x_{i}\right]-\rho \int A \wedge \prod_{i} x_{i}, \\
= & \left\{S_{\mathrm{FS}}\left[\psi, A, x_{i}\right]-\frac{V_{\mathrm{F}}}{(2 \pi)^{d-1}} \int A \wedge \prod_{i} x_{i}\right\} \\
& -\left(\rho-\frac{V_{\mathrm{F}}}{(2 \pi)^{d-1}}\right) \int A \wedge \prod_{i} x_{i} .
\end{aligned}
$$

The collection in $\{\ldots\}$ is gauge invariant but the last term is not if $\left(\rho-V_{\mathrm{F}} /(2 \pi)^{d-1}\right) \notin \mathbb{Z}$. We should therefore view the last term as a polarization term in $d$ space dimensions, hence Eq. (18). The lesson is that bulk polarization does not directly give a boundary charge density, rather it leads to a boundary quantum anomaly. In Appendix F, we numerically study a free fermion model on square lattice and verify that Eq. (18) is always satisfied across a range of parameters with qualitatively different edge behaviors. Equation (18) also applies with $\mathbf{P}$ replaced by $\tilde{\mathbf{P}}$ if we also replace the bound charge density by the excess charge density.

When viewed as an anomaly-matching condition, Eq. (18) can also be applied to surface states other than Fermi liquidswe simply need to replace $V_{\mathrm{F}}$ by the appropriate anomaly indicators of the low-energy theories. Namely, we demand $\rho=n_{A}+P \bmod 1$ where $n_{A}$ is the anomaly indicator of the low-energy effective theory. For example, for rational values of $\rho-P$, the anomaly can be matched by a gapped GS with intrinsic topological order, which typically hosts nontrivial quasiparticles with fractional electric charge. In such states, the anomaly is encoded in how the topologically nontrivial excitations (like anyons in 2D and flux loops in 3D) transform under translation symmetries. These anomalies are closely related to the LSM-type theorems that constrain the possible low-energy theories of a given lattice system [19,37-42]. We briefly describe the LSM-type anomaly for topological orders in two and three dimensions in Appendix E. These results reduce to the previously obtained boundary Luttinger relations in the absence of polarization, as described in Ref. [43], and also apply to 2D systems with a nonzero Hall conductivity, provided an appropriate gauge choice in Appendix F, discussed previously in Ref. [44] within band theory.

The logic we used to study the boundary can also be used to study dislocations. A dislocation has space dimension $(d-2)$ and therefore can preserve at most $(d-2)$ translation symmetries. For simplicity, we consider a dislocation with Burgers vector $\mathbf{B}=\widehat{x}_{2}$ and unbroken translation symmetries $\mathbb{Z}^{x_{3}} \times \ldots \mathbb{Z}^{x_{d}}$. The polarization term Eq. (8) reduces to the following on the dislocation (with space-time dimension $d-1$ ):

$$
S_{\text {disloc }}=P_{1} \int A \wedge x_{3} \wedge \ldots x_{d} .
$$

This has the same form as the boundary term Eq. (17), only in one dimension lower. This term leads to the same Luttinger theorem violation as Eq. (18) on the $(d-2)$-dimensional dislocation, where $V_{\mathrm{F}}$ is again interpreted as the anomaly indicator of the low-energy effective theory. A special case is $d=2$ which has been discussed in Ref. [45], where the $V_{\mathrm{F}}$ term is not needed and the polarization directly determines the fractional electric charge nucleated at the dislocation point.

\section{SUMMARY}

In this paper, we proposed a nonperturbative definition of the physically measurable polarization density in a crystalline insulator through translation properties of test magnetic monopoles. Our formalism is applicable in any space dimension to systems of interacting electrons but equally to interacting bosons or spins that enjoy a U(1) symmetry, as long as there is a unique gapped GS. The central result is a response involving background $\mathrm{U}(1)$ fluxes and translation gauge fields, captured by a topological term. This response is topological despite the fact that the coefficient which is identified with the polarization is not quantized. Indeed, to probe this response, one needs to implement a global (nonperturbative) change, e.g., a U(1) flux (monopole/instanton), a lattice shear, a dislocation, or a physical boundary. This surprising connection seems natural in light of the necessity of charge quantization to properly define polarization [13], which is the consequence of a compact $\mathrm{U}(1)$ symmetry group, which, by the Dirac quantization argument, is related to the existence of magnetic monopole operators. The subtleties in previous literature associated with defining polarization ( $\mathbf{P}$ vs $\tilde{\mathbf{P}}$ ) are neatly accounted for by gauge field configurations that have different distributions within a unit cell, corresponding to different approaches toward the continuum limit. Besides being given a recipe to obtain the polarization in numerical calculations, the connection bears conceptual significance to 
boundary physics. We see that the classical relation between polarization and boundary charge density receives a quantum correction in the form of an anomaly associated with the boundary low-energy theory. For a boundary Fermi liquid, the anomaly is associated with the familiar Luttinger theorem and, for a more general boundary phase, it is associated with a LSM-like theorem, but for a general filling.

Note added: We note recent works [26] that studied the response of $(2+1) \mathrm{D}$ Abelian topological phases with crystalline symmetries utilizing crystalline gauge fields for translations and rotations.

\section{ACKNOWLEDGMENTS}

We gratefully acknowledge helpful discussions with G. Baskaran, Jing-Yuan Chen, Meng Cheng, Gil Young Cho, Dominic Else, Davide Gaiotto, Charlie Kane, T. Senthil, YiZhuang You, and Liujun Zou. A.V. was supported by a Simons Investigator award and by a grant from the Simons Foundation (Grant No. 651440). Research at Perimeter Institute (Y.-C.H. and C.W.) is supported by the Government of Canada through the Department of Innovation, Science and Economic Development Canada and by the Province of Ontario through the Ministry of Research, Innovation and Science.

\section{APPENDIX A: POLARIZATION WITH NONTRIVIAL UNIT CELL STRUCTURES}

Here we discuss different definitions of polarization to emphasize the lattice point of view. Most of the physics below are discussed in Refs. [11,16].

To illustrate the essential point, it suffices to consider a simple 1D lattice with two sublattices and one electron orbital on each-generalizations to more complicated unit cells (or even higher dimensions) will be straightforward. We label the unit cells by $i \in \mathbb{Z}$ and sublattices by $\{a, b\}$. We consider a simple insulator with unit charge occupation per unit cell, namely, $\left\langle n_{i, a}+n_{i, b}\right\rangle=1$, with an extra unit negative (ion) charge $q_{\text {ion }}=-1$ sitting on site $a$ to make the entire system charge neutral.

The electric polarization is defined via the response to a smooth gauge field $(\varphi, A)$ defined on the lattice-but what does smooth mean? Clearly we want the gauge field to be slowly varying when moving from one unit cell to another, which means the lattice momentum of the gauge field is close to zero. For example, $A_{i, a ; i, b} \approx A_{i+1, a ; i+1, b}$ and $\varphi_{i, a} \approx \varphi_{i+1, a}$. However, this does not uniquely specify how the gauge field should be distributed within a unit cell, namely, how $A_{i, a ; i, b}$ should be compared with $A_{i, b ; i+1, a}$, or how $\varphi_{i, a}$ should be compared with $\varphi_{i, b}$. Apparently, we have a choice to make here. One simple choice is to demand $A_{i, a ; i, b}=0$ and $\varphi_{i, a}=$ $\varphi_{i, b}$. The continuum limit of the gauge fields (now a smooth function of the continuum coordinate $x$ with no explicit dependence on the sublattice index) will be $A(x=i)=A_{i, b ; i+1, a}$ and $\varphi(x=i)=\varphi_{i, a}$. This is equivalent to viewing the entire unit cell as a single point in space. The polarization defined via the response to such field configurations effectively measures only the interunit cell entanglement and is called $\tilde{\mathbf{P}}$ in Ref. [11]. We can make a more general choice as follows: We demand

$$
\begin{aligned}
(1-\alpha) A_{i, a ; i, b} & =\alpha A_{i, b ; i+1, a}, \\
(1-\alpha)\left(\varphi_{i, b}-\varphi_{i, a}\right) & =\alpha\left(\varphi_{i+1, a}-\varphi_{i, b}\right),
\end{aligned}
$$

for some constant $\alpha$. The continuum limit is then

$$
\begin{aligned}
& A(x=i) \equiv \alpha A_{i, a ; i, b}+(1-\alpha) A_{i, b ; i+1, a}, \\
& \varphi(x=i) \equiv \varphi_{i, a} .
\end{aligned}
$$

The physical meaning is also clear: Wwe interpret the two sublattices $a, b$ as separated by a distance $\alpha$ in real space (with lattice unit normalized to unity). If the lattice system originates from a continuum with the two sites separated physically by a distance $\alpha$, then this choice corresponds to a physically uniform electric field, and therefore produces the polarization $\mathbf{P}$ for a uniform electric field. To obtain the polarization difference $P-\tilde{P}$, consider the lattice-scale action difference $S\left[A_{P}^{\mu}\right]-S\left[A_{\tilde{P}}^{\mu}\right]$, where $A_{P}^{\mu}, A_{\tilde{P}}^{\mu}$ are the corresponding lattice gauge field distributions with the same continuum limit $A^{\mu}(x, t)$. Using the definition of $A_{P}^{\mu}$ in Eqs. (A1) and (A3) and the fact that $j_{i, a ; i, b}-j_{i, b ; i+1, a}=\partial_{t} n_{i, b}$, where $j$ is the lattice current operator that couples to the $A$ field, one can see that

$$
\begin{aligned}
S\left[A_{P}^{\mu}\right]-S\left[A_{\tilde{P}}^{\mu}\right]= & \sum_{i} \int d t \alpha n_{i, b}\left[\partial_{t} A(x=i)\right. \\
& -(\varphi(x=i+1)-\varphi(x=i))],
\end{aligned}
$$

which simply means that $\mathbf{P}-\tilde{\mathbf{P}}=\alpha\left\langle n_{i, b}\right\rangle$, and can be interpreted as a classical contribution, namely a charge $q_{b}$ sitting at site $b$ contributes a dipole moment $\alpha q_{b}$. Different choices of the intracell gauge field distribution also lead to different definitions of charge density (per unit cell) in the continuum limit. In the continuum limit, we define charge density as $\rho(x)=\delta S / \delta \varphi(x)$ where $\varphi(x)$ is in the continuum limit as discussed above. This is easy for the gauge field probing $\tilde{\mathbf{P}}$, where $\varphi$ is unique within a unit cell, and we simply get $\rho_{i}=\left\langle n_{i, a}+\right.$ $\left.n_{i, b}\right\rangle+q_{\text {ion. }}$. However, with nontrivial $\alpha$ (as for the standard $\mathbf{P}$ ), there is a correction from the nonuniformity of $\varphi$ within the unit cell. A simple calculation gives $\delta \rho_{i}=-\alpha \nabla\left\langle n_{i, b}\right\rangle$, which is nonzero only at the boundary. The relation $\rho=-P(\bmod$ 1 ) at the boundary (an insulating boundary in $d>1$ ) holds for both $P$ and $\tilde{P}$ as long as the corresponding definitions for charge density are used. A similar difference between current operators from $j(x)=\delta S / \delta A(x)$ in different continuum limits was also discussed in the literature [11].

The above discussion implies that when one considers small momentum (long wavelength) components of $d A$, e.g., a uniform electric field induced by monopole translation, Eq. (8) gives a response controlled by the conventional polarization $\mathbf{P}$; while $d A$ around higher momenta $n_{i} \mathbf{G}_{i}\left(n_{i} \in \mathbb{Z}\right)$ probes sites inside a unit cell with different weights. In one extreme case, where one concentrates inductive electric field on only the intercell bond, the response term gives $\tilde{\mathbf{P}}$.

\section{APPENDIX B: DETAILS OF 2D CALCULATION}

\section{Recipes for calculation}

Consider a square lattice with $L_{x} \times L_{y}$ unit cells, and assume a unique gapped GS. We put a total magnetic flux of 
$2 \pi$ uniformly on the entire surface. To be concrete, let us take the following gauge (analogue of Landau gauge on a discrete torus):

$$
\begin{aligned}
A_{i, i+\widehat{x}} & =-\frac{2 \pi y}{L_{y}} \delta_{x, L_{x}-1}, \\
A_{i, i+\widehat{y}} & =\frac{2 \pi x}{L_{x} L_{y}} \\
i & =(x, y), x \in\left\{0, \ldots L_{x}-1\right\}, y \in\left\{0, \ldots L_{y}-1\right\} .
\end{aligned}
$$

In this gauge, a unit translation in $\widehat{x}$ (denoted $T_{x}$ ) should be followed by a gauge transform that acts nontrivially only on the $x=0$ strip,

$$
G_{x}=\exp \left(-i \sum_{i} \frac{2 \pi y}{L_{y}} \delta_{x, 0} \widehat{q_{i}}\right),
$$

where $\widehat{q}_{i}$ is the charge density operator on site $i$. The $\widehat{y}$ translation (denoted $T_{y}$ ), in contrast, does not need an additional gauge transform.

Strictly speaking, however, on a finite torus neither $G_{x} T_{x}$ nor $T_{y}$ is a true symmetry since the Wilson loop along the nontrivial $\widehat{y}$ and $\hat{x}$ cycles cannot be translationally invariant under $T_{x}$ and $T_{y}$, respectively. As we can see explicitly from Eq. (B1), the Wilson loops change by $\int d y \delta A_{y}=-2 \pi / L_{x}$ on every $\widehat{y}$ cycle and $\int d x \delta A_{x}=2 \pi / L_{y}$ after $T_{x}$ and $T_{y}$, respectively. This noninvariance of Wilson loops cannot be cured by a gauge transform. To overcome this issue, we consider modified translations $\tilde{T}_{x}=F_{y} G_{x} T_{x}$ and $\tilde{T}_{y}=F_{x} T_{y}$, where $F_{y}, F_{x}$ are adiabatic evolutions that modify the $A$ fields by $\delta A$ at the end of the evolutions, where

$$
\begin{aligned}
& F_{y}: \quad \delta A_{x}=0, \quad \delta A_{y}=\frac{2 \pi}{L_{x} L_{y}}, \\
& F_{x}: \quad \delta A_{x}=-\frac{2 \pi}{L_{y}} \delta_{x, L_{x}-1}, \quad \delta A_{y}=0 .
\end{aligned}
$$

The composite operations $\tilde{T}_{x}, \tilde{T}_{y}$ preserve the Hamiltonian, and therefore produce well-defined Berry phases $\phi_{x}, \phi_{y}$ which we identify with $\mathbf{k}_{e}=-\mathbf{k}_{\mathcal{M}}=2 \pi\left(P_{y},-P_{x}\right)$.

The connection between the monopole momentum and polarization can also be understood from the structure of $\tilde{T}_{x}, \tilde{T}_{y}$. Consider the operations $\tilde{T}_{x}^{L_{x}}$ and $\tilde{T}_{y}^{L_{y}}$. Using $T_{x}^{L_{x}}=T_{y}^{L_{y}}=1$, one can see that the two operations become the familiar adiabatic $2 \pi$ flux threading in the $\widehat{y}$ and $-\widehat{x}$ directions, respectively. The corresponding Berry phases are $\left(\Phi_{x}, \Phi_{y}\right)=$ $2 \pi\left(L_{x} P_{y},-L_{y} P_{x}\right)$, in agreement with our previous result. Notice that since $\tilde{T}_{i}^{L_{i}} \neq 1$, the translation Berry phase defined above is not quantized on a finite system-this is consistent with the fact that polarization can take continuous value in a finite system.

In practice, since $F_{x}, F_{y}$ only threads a small flux of order $O(1 / L)$, one would expect their actual effect to be small, especially at large $L$. One can then consider the simpler amplitudes $\left\langle\Omega\left|G_{x} T_{x}\right| \Omega\right\rangle$ and $\left\langle\Omega\left|T_{y}\right| \Omega\right\rangle(|\Omega\rangle$ being the GS in the flux background). These will have magnitudes smaller than one on a finite torus, but as long as it is nonvanishing (in fact, we expect it to approach unity in the thermodynamic limit), one can extract the phase of the amplitude, and this phase should give the monopole momentum, which in turn gives the polarization density. More explicitly,

$$
\left\langle\Omega\left|G_{x} T_{x}\right| \Omega\right\rangle=\rho_{x} e^{2 \pi i P_{y}}, \quad\left\langle\Omega\left|T_{y}\right| \Omega\right\rangle=\rho_{y} e^{-2 \pi i P_{x}},
$$

where $\rho_{x, y}$ are magnitudes that are nonvanishing in the thermodynamic limit (in practice they $\rightarrow 1$, see Sec. . Equation (B5) is in the same spirit with Resta's formula [17] for polarization in $1 \mathrm{D}$, which is the phase of the (smaller than one) amplitude $\left\langle\Omega\left|\exp \left(i x \widehat{q}_{x} / L\right)\right| \Omega\right\rangle$. In higher dimensions, Resta's amplitude vanishes in the thermodynamic limit and cannot be used to extract polarization [11]. Our prescription using the amplitudes $\left\langle\Omega\left|G_{x} T_{x}\right| \Omega\right\rangle$ and $\left\langle\Omega\left|T_{y}\right| \Omega\right\rangle$ can be viewed as a proper generalization of Resta's formula to $2 \mathrm{D}$. In fact, this prescription has been carried out in previous studies of monopoles in 2D U(1) spin liquids [32].

Strictly speaking, our recipe gives the polarization of the GS in the $2 \pi$-flux background $|\Omega\rangle$, which is slightly different from the original GS without the flux $|\Omega\rangle_{0}$. The two should agree in the thermodynamic limit. To see this, let us consider insulators with zero Hall conductance. If there is no symmetry other than charge conservation and translations, the leading order term in the response theory that can cause a magnetic flux to change the polarization is $\Delta \mathcal{L} \sim \alpha_{i} B E_{i}$ for some constants $\alpha_{i}(i=x, y)$. This means that a total $2 \pi$ flux will change polarization by $O(B) \sim O\left(1 / L^{2}\right)$. With time-reversal symmetry, the leading order term becomes $\sim B^{2} E$ and the change of polarization in the $2 \pi$-flux background becomes $O\left(B^{2}\right) \sim O\left(1 / L^{4}\right)$. This error will likely be dominated by other finite-size effects such as omitting the flux-threading $F_{x, y}$ in the calculation. This argument is reliable for insulators without Hall conductance, since we expect all terms in the response theory to be local and manifestly gauge invariant.

If the unit cell contains two neighboring sites in the $x$ direction, i.e., $L_{x}$ even and a unit cell contains $(2 n, m),(2 n+$ $1, m)(n, m \in \mathbb{Z})$, the above recipe only distributes a nonvanishing $A$ field on bonds between unit cells of choice, and $A$ vanishes within each unit cell, which corresponds to calculating $\tilde{\mathbf{P}}$ in Ref. [11]. The actual unit cell structure and geometry do not contribute to monopole translation properties, or polarization, obtained in such ways. In general, the polarization and the monopole momentum depend on the choice of unit cells.

To obtain the polarization $\mathbf{P}$, with both intra(classical) and intercell effects, we give a recipe to account for the unit cell geometry, applicable to generic systems. To this end, we first give a continuum function for gauge field $\mathbf{A}$ on the torus which can then be used to determine the discrete gauge fields. Taking the distance between neighboring unit cells to be 1 and the Bravais lattice to be square, the continuum gauge field reads

$$
\begin{aligned}
& A_{x}(x, y)= \begin{cases}0 & 0 \leqslant x<L_{x}-1 \\
-\frac{2 \pi y}{L_{y}} & L_{x}-1 \leqslant x<L_{x},\end{cases} \\
& A_{y}(x, y)= \begin{cases}\frac{2 \pi x}{L_{x} L_{y}} & 0 \leqslant x \leqslant L_{x}-1 \\
\frac{2 \pi\left(L_{x}-1\right)}{L_{x} L_{y}}\left(L_{x}-x\right) & L_{x}-1<x<L_{x} .\end{cases}
\end{aligned}
$$

Note the function is not single valued but is well defined and hence poses no problems for obtaining the gauge fields on the discrete lattice. When put on the lattice, the gauge connection on one bond $l$ is given by $\int_{l} d \mathbf{x} \cdot \mathbf{A}(\mathbf{x})$, i.e., the line integral of continuum $\mathbf{A}$ along the bond. 
Once put on a lattice, the flux close to the slit at $y=$ $L_{y}, L_{x}-1 \leqslant x \leqslant L_{x}$ should have an $O(1)$ deviation from $2 \pi /\left(L_{x} L_{y}\right)$ due to the discontinuity in Eqs. (B5) (the total flux threading the unit cell at $\left(L_{x}-1, L_{y}-1\right)$ is hence $\left.2 \pi /\left(L_{x} L_{y}\right)-2 \pi\right)$. One could compensate for this deviation by altering the gauge connection on bonds inside the slit, such that the deviation is concentrated to a set of elementary plaquettes (i.e., not containing any smaller plaquettes) that contain the point $\left(L_{x}, L_{y}\right)$, whose flux equals $2 \pi /\left(L_{x} L_{y}\right) A-$ $2 \pi$ ( $A$ is the area of the elementary plaquette). This fixes the translation symmetry breaking of flux derived from the continuum recipe. Upon translation $T_{y}$, one carefully performs a gauge transform on sites in the slit $G_{y}$ to restore the gauge connection as much as possible, the amplitude of $\left\langle\Omega\left|G_{y} T_{y}\right| \Omega\right\rangle$ is comparable to unity; the phase converges in thermodynamic limit to the conventional polarization $\mathbf{P}$.

The two recipes have the same flux configuration on torus and hence are connected by a gauge transform. However, this gauge transform generally does not commute with $G_{y} T_{y}$ and will change the momentum obtained, consistent with getting $\tilde{\mathbf{P}}$ versus $\mathbf{P}$ for the two recipes. For example, in the above square lattice model, we assume a unit cell at $(n, m)$ contains two sites at $(n, m),(n+1 / 2, m)$, respectively [in notation of Eq. (B1), the coordinates read $(2 n, m),(2 n+1, m)]$. Then the two recipes built upon Eqs. (B1) and (B6) differ by a gauge transform on sites with $x=L_{x}-1 / 2$ by the operator $e^{-i \sum_{y} \frac{\pi y}{L_{y}} \widehat{\rho}\left(L_{x}-1 / 2, y\right)}$. From the commutation relation between this gauge transform and the $G_{y} T_{y}$ operation, one can see that the change of momentum from the gauge transform as $L \rightarrow \infty$ is precisely $\delta k_{y}=-\pi\left\langle\widehat{\rho}\left(L_{x}-1 / 2, y\right)\right\rangle$, which leads to a change in polarization $P_{x}-\tilde{P}_{x}=\left\langle\widehat{\rho}\left(L_{x}-1 / 2, y\right)\right\rangle / 2$, in agreement with the intuition that the difference between $\mathbf{P}$ and $\tilde{\mathbf{P}}$ can be seen as a classical dipole moment within the unit cell.

\section{Review of band theory calculation}

For a $d$-dimensional lattice system with translation symmetries and periodic boundary conditions in all directions, the polarization corresponds intuitively to the dipole moment in each unit cell. For free fermions, the polarization contributed by an occupied band is given by the integrated Berry connection (the Wilson loop) in the Brillouin zone [13],

$$
\mathbf{P}=\int_{\mathrm{BZ}} \frac{d^{d} \mathbf{k}}{(2 \pi)^{d}}\left\langle u_{\mathbf{k}}\left|i \partial_{\mathbf{k}}\right| u_{\mathbf{k}}\right\rangle \quad(\bmod 1),
$$

where $\left|u_{\mathbf{k}}\right\rangle$ is the periodic part of the Bloch state at momentum $\mathbf{k}$ and the integration is taken over the entire Brillouin zone. (Here $u_{\mathbf{k}}(r)=e^{-i \mathbf{k} \cdot \mathbf{r}} \psi(r)$.) We also discuss $\tilde{\mathbf{P}}$ [11] if we instead use $\tilde{u}_{\mathbf{k}}(\mathbf{r})=e^{-i \mathbf{k} \cdot \mathbf{R}} \psi(\mathbf{r})$, where $\mathbf{r}=\mathbf{R}+\mathbf{r}_{i}$ and $\mathbf{R}$ is the Bravais lattice vector associated with $\mathbf{r} .\left[\tilde{u}_{\mathbf{k}}(\mathbf{r})=\tilde{u}_{\mathbf{k}+\mathbf{G}_{i}}(\mathbf{r})\right.$. $]$

In the presence of gapless Dirac cones, the band theory polarization Eq. (B6) is not uniquely defined. This ambiguity can also be understood from monopole momentum: In a $2 \pi$ flux background there are fermion zero modes associated with the Dirac fermions, and filling different zero modes gives different GSs, with different total momenta. We now discuss this within the usual band theory formulation. For concreteness, consider a system of spin-1/2 fermions forming two Dirac valleys, say, at momenta $\mathbf{K}, \mathbf{K}^{\prime}$. The Wilson loop for each spin $\alpha$ in the $\widehat{k}_{1}$ direction

$$
\mathcal{P}_{\alpha}\left(k_{2}\right)=\int \frac{d k_{1}}{2 \pi}\left\langle u_{\alpha, \mathbf{k}}\left|i \partial_{k_{1}}\right| u_{\alpha, \mathbf{k}}\right\rangle
$$

has a discontinuity of $\pm \pi$ when $k_{2}$ passes through $K_{2}$ and $K_{2}^{\prime}$. The polarization

$$
P_{1}=\sum_{\alpha=\uparrow, \downarrow} \int \frac{d k_{2}}{2 \pi} \mathcal{P}_{\alpha}\left(k_{2}\right)
$$

requires a choice of the jump in $\mathcal{P}_{\alpha}(\pi$ or $-\pi)$ at each Dirac point. For the polarization to be gauge-invariant, $\sum_{\alpha} \mathcal{P}_{\alpha}\left(k_{2}\right)$ should be single-valued in the entire Brillouin zone (i.e., no net Chern number). This leads to six different choices of the jumps in $\mathcal{P}_{\alpha}$ at the Dirac points. Now, from the monopole momentum point of view, in a $2 \pi$-flux background there are four zero modes (one from each Dirac cone) and gauge-invariance requires the GS to fill half of the zero modes, which leads to $C_{2}^{4}=6$ different choices-in exact agreement with the band theory consideration.

\section{An example}

Our numerical prescription for calculating polarization density through amplitudes like $\left\langle\Omega\left|T_{y}\right| \Omega\right\rangle(|\Omega\rangle$ being the many-body GS in the presence of a uniform $2 \pi$ flux background) is well-defined for generic many-body systems. In the special case of free fermions, we expect our prescription to agree with the band theory results from Eq. (B6). We demonstrate this through an example of a Dirac semimetal (with a specific choice of zero-mode fillings). We consider a square lattice, labeled by two orthogonal unit lattice vectors $e_{1,2}$, with two orbitals and two spin species on each site. The Hamiltonian for our spin-1/2 fermions reads

$$
\mathcal{H}=\sum_{\langle i j\rangle, \alpha, \beta} t_{i \alpha, j \beta} e^{i a_{i j}} f_{j \beta, s}^{\dagger} f_{i \alpha, s}+\sum_{i, \alpha, \beta} t_{i \alpha, i \beta} f_{i \beta, s}^{\dagger} f_{i \alpha, s},
$$

where $s=\uparrow, \downarrow$ labels spin indices, $\alpha, \beta=1,2$ label orbitals, $\langle i j\rangle$ denotes neighboring or sites linked by a diagonal bond, and hopping amplitudes read

$$
\begin{aligned}
t_{\left[l_{1}, l_{2}\right],\left[l_{1}+1, l_{2}\right]} & =1, \\
t_{\left[l_{1}, l_{2}\right],\left[l_{1}, l_{2}+1\right]} & =(-1)^{l_{1}}, \\
t_{\left[l_{1}-1, l_{2}\right],\left[l_{1}, l_{2}-1\right]} & =(-1)^{l_{1}} t \quad(t \in[0,1]), \\
t_{\left[l_{1}, l_{2}\right]\left[l_{3}, l_{4}\right]} & =t_{\left[l_{3}, l_{4}\right]\left[l_{1}, l_{2}\right]},
\end{aligned}
$$

where we have relabeled subscripts $i \alpha$ by $\left[l_{1}, l_{2}\right]$ through $l_{1}=2 * i_{1}+\alpha, l_{2}=i_{2}$ ( site $i$ with coordinates $\left(i_{1}, i_{2}\right)$ in $e_{1,2}$ basis) and $t$ is the tuning parameter. Hopping amplitudes on other diagonal or neighboring bonds not covered in Eq. (B10) vanish. The two limits $t=0,1$ correspond to a square with $C_{4}$ rotation and an effective triangular lattice with $C_{6}$ rotation, respectively. Diagonalizing this Hamiltonian in momentum space gives gapless dispersion at half-filling. To avoid the ambiguity for a Wilson loop operator when crossing Dirac fermions as discussed in Appendix (B10), we stipulate the two bands for spin up/down has Chern number \pm 1 , respectively, i.e., effectively open an infinitesimal quantum spin hall mass. For the monopole momentum calculation, the gauge connection on links $a_{i j}$ analogous to Landau gauge in Eq. (B1) gives 
a total flux of $2 \pi$ and the quantum spin hall mass indicates that one fills only two zero modes of one spin species, giving a monopole carrying spin 1 . Figure 1 in the main text shows a comparison of polarization $P_{1}$, calculated numerically using Eq. (B6) long the direction of reciprocal vector for $e_{1}$ and the monopole momentum $k_{2}$ along the orthogonal $e_{2}$ direction, calculated as in Appendix B 1 as one tunes $t$ from 0 to 1. The polarization obtained from Eq. (B6) is discretized as a summation of the Berry phase $\ln \left\langle u_{\mathbf{k}} \mid u_{\mathbf{k}+\epsilon}\right\rangle$ for 30000 points in the Brillouin zone; the momentum is calculated on a lattice of linear size $L=50$. For the special cases when $t=0,1$ the momentum $k_{2}=\pi, 2 \pi / 3$ agrees with results in Refs. [32,46].

\section{APPENDIX C: POLARIZATION AND OTHER TOPOLOGICAL QUANTITIES}

In $2 \mathrm{D}$, the polarization density is not invariant under large gauge transforms if the system has a nonzero Hall conductance $\sigma_{x y} \neq 0$. This is known in band theory, where Eq. (B6) is not invariant under large gauge transforms in real space-in fact, this is one way to define integer quantum Hall effect within band theory. Beyond band theory, it is also easy to understand why this is so from the monopole momentum: A $2 \pi$-flux induces an extra charge $\delta Q=\sigma_{x y}$ in the GS, which makes the total momentum noninvariant under large gauge transforms. The total polarizations $P_{x} L_{y}$ and $P_{y} L_{x}$ are still well-defined (gauge invariant) mod 1. Similarly, if the system forms a quantum spin Hall insulator, with a nonzero $S_{z}$ spin trapped in a magnetic flux unit, then the polarization is not invariant under a large $S_{z}$-gauge transform. In all such cases, the polarization remains meaningful (unambiguisly defined) for a given gauge if the gauge field remains nondynamical.

Contrary to the Hall conductance, a nonzero magnetoelectric angle $\Theta$ in $3 \mathrm{D}$,

$$
\frac{\Theta}{4 \pi^{2}} \mathbf{E} \cdot \mathbf{B}
$$

does not obstruct the gauge invariance of polarization density. Within band theory, the $\Theta$ angle can be interpreted as the magnetoelectric polarizability $[47,48]$, i.e., a magnetic field induces an extra polarization density:

$$
\Delta \mathbf{P}=\frac{\Theta}{4 \pi^{2}} \mathbf{B} .
$$

The monopole point of view provides a simple understanding of the above relation beyond band theory: When $\Theta \neq 0$, the monopole traps a fractional charge $q=\Theta / 2 \pi$ and becomes a dyon [49]. When a magnetic field is turned on, say, in $\widehat{z}$, the monopole also sees the field due to the fractional $q$. This contributes to the noncommutativity of $T_{x}$ and $T_{y}$, with the additional phase factor given by $q B=(\Theta / 2 \pi) B$. Using Eq. (16) as the definition of polarization, we immediately obtain Eq. (C2).

\section{APPENDIX D: ANOMALY FROM A FERMI SURFACE}

In this Appendix, we derive the anomaly term Eq. (20) which proves the Luttinger theorem in any spatial dimension $d$. The logic is to partition the Fermi surface into infinitesimally small patches in whose proximity reside chiral fermions, that effectively live in $(1+1) d$. The chiral anomaly from each of these fermions adds up to give Eq. (20).

Let us first write down the anomaly for a 1D chiral fermion with the free Hamiltonian $\psi^{\dagger} i\left( \pm \partial_{x}\right) \psi$, where we set velocity to unity and \pm represents right (left) movers under consideration. Next we couple to theory to both a U(1) electromagnetic field $A$ and an $x$-translation gauge field (elasticity tetrad) $x$. The momentum of the chiral fermion $k_{\mathrm{F}}$ becomes the coupling constant between the translation gauge field $x$ and the fermion (in analogy to electric charge $e$ as the coupling constant between EM field $A$ and a fermion, here the charge of translation-momentum-mediates the coupling). Hence the covariant derivative $i \partial_{x, t} \rightarrow i \partial_{x, t}+A_{x, t}+k_{\mathrm{F}} x_{x, t}$ where the subscript denotes the space-time component of the $1-$ form gauge field, omitted hereafter. To obtain the mixed anomaly between $A, x$, one goes to one higher dimension $(2+1) d$ bulk of the chiral fermion-a quantum Hall insulator with Chern number $C= \pm 1$, with the low-energy topological quantum field theory action:

$$
S=\frac{ \pm 1}{4 \pi} \int\left(A+k_{\mathrm{F}} x\right) \wedge d\left(A+k_{\mathrm{F}} x\right) .
$$

Note that since we introduce elasticity tetrads in addition to $A$, the familiar Chern-Simons term $A \wedge d A$ is modified as such. The mixed term in Eq. (D2) reads $\pm \int \frac{k_{\mathrm{F}}}{2 \pi} x \wedge d A$, from which descends a boundary term $\mp \int \frac{k_{\mathrm{F}}}{2 \pi} A \wedge x$.

Now that we have the desired $(1+1) d$ anomaly, consider in a $d$ space dimension system, compactify $(d-1)$ dimensions and derive similar anomaly for the effective $(1+1) d$ system along the remaining $i$ th primitive lattice vector direction. We inspect a small patch on the Fermi surface with momentum range $\left(k_{1} \pm \delta k_{1} / 2, \cdots k_{i}, \cdots k_{d} \pm \delta k_{d} / 2\right)\left(\delta k_{j} \geqslant\right.$ 0 ,the variation of $k_{i}$ is neglected to zeroth order of the anomaly). On such a patch with an area $\Delta s_{i}=\prod_{j \neq i} \delta k_{j}$, there are $\left(\frac{\Delta s_{i}}{(2 \pi)^{d-1}} \prod_{j \neq i} L_{j}\right)$ chiral fermions along the $i$ th direction, each associated with an anomaly $\frac{\mp k_{i}}{2 \pi} \int A \wedge x_{i}$ ( $\mp$ in numerator results from right (left) movers given by the orientation of the small patch projected onto $i$ th reciprocal vector direction). Adding all patches up, the self-Chern-Simons terms vanish and the remaining anomaly reads

$$
\begin{array}{r}
S_{\mathrm{FSanomaly}}=-\frac{\prod_{j \neq i} L_{j}}{(2 \pi)^{d}} \sum_{\mathrm{FS}} \Delta s_{i} \eta_{i} k_{i} \int A \wedge x_{i} \\
\rightarrow-\frac{V_{\mathrm{F}}}{(2 \pi)^{d}} \int A \wedge \prod x_{i} .
\end{array}
$$

where $\sum_{\mathrm{FS}}$ counts all patches on the Fermi surface, $\eta_{i}= \pm 1$ denotes the orientation of each patch along/against $i$ th reciprocal lattice vector, and we use the identity on Luttinger volume $\sum_{\mathrm{FS}} \Delta s_{i} \eta_{i} k_{i}=V_{\mathrm{F}}$. The second line arises after we introduce translation gauge fields along the other $(d-1)$ directions and the numerator in the first line: $\prod_{j \neq i} L_{j} \rightarrow$ $\int \wedge \prod_{j \neq i} x_{j}$. The final result puts all $x_{i}$ 's on equal footing and hence it correctly captures the anomaly of the Fermi surface under large gauge transforms along any spatial directions. Adding the anomaly term to the Fermi surface theory will make the full theory anomaly-free, as promised in Eq. (21). 


\section{APPENDIX E: LSM ANOMALY INDICATORS FOR TOPOLOGICAL ORDERS}

First, we review an important notion for a topological order with a global U(1) symmetry in general $d$ dimensions known as the fluxon. Consider an instanton of the $A$ field, which is an operator supported on a $(d-2)$ dimensional submanifolds in space, with $\int d A=2 \pi$ on the two complementary spatial dimensions. For $d=2$, it is a point flux insertion and for $d=3$ it is a unit flux loop. Dirac quantization requires this object to be unobservable from far away. However, in a topologically ordered state, there can be nontrivial quasiparticles that carry fractional electric charge, and moving these fractional charges around the instanton will naively produce an observable Aharanov-Bohm phase. The resolution is that the bare instanton is attached with another nontrivial excitation, called the fluxon, from the topological order. The property of the fluxon is such that the combined object becomes unobservable from far away. For example, a fractionally charged quasiparticle will have a nontrivial braiding phase with the fluxon so it braids trivially with the combination of fluxon and bare instanton. In 2D, the fluxon is an anyon excitation and in $3 \mathrm{D}$ it is a loop excitation.

In general, anomalies involving a U(1) global symmetry in topological quantum field theories are encoded in the properties of fluxons. Essentially, if the fluxon carries a fractional quantum number under other symmetries, in our case lattice translations, then the instanton will also carry the fractional symmetry quantum numbers since it is bound with a fluxon. Since the instanton is supposed to be unobservable, this becomes an anomaly. The fluxon has space-time dimension $d-$ 1 , and crystal symmetry fractionalization can be described using a partition function in a $d$ space-time dimension:

$$
\mathcal{L}_{\text {fluxon }}=-n_{A} \int x_{1} \wedge x_{2} \ldots \wedge x_{d},
$$

for which the fluxon lives on the boundary of the $d$ dimensional (space-time) manifold, and $n_{A} \in[0,1)$ is the LSM anomaly indicator.

At $d=2$, the fluxon is an (Abelian) anyon particle, and Eq. (E1) means that the fluxon transforms projectively under translation symmetries:

$$
T_{2}^{-1} T_{1}^{-1} T_{2} T_{1}=e^{-i 2 \pi n_{A}} .
$$

This relation has been discussed in Ref. [50]. We note that this result is equally applicable for magnetic translation symmetries, where a nontrivial $\mathrm{U}(1)$ flux $\phi$ is enclosed in each unit cell. As a simple example, consider a shortrange entangled integer quantum Hall state. The fluxon in this case must be an integer multiple of the local electron since there is no fractional excitation. Specifically, to make the $2 \pi$-flux unobservable, the fluxon must carry electric charge $-2 \pi \sigma_{x y}$, namely, it is the bound state of $-2 \pi \sigma_{x y}$ electrons. The effective magnetic flux seen by this fluxon is therefore $-2 \pi \phi \sigma_{x y}$. To satisfy Eq. (E2), we must therefore have

$$
\phi \sigma_{x y}=n_{A}(\bmod 1) .
$$

This relation has also been discussed in Ref. [50].
At $d=3$, the fluxon is a loop excitation with finite tension. Equation (E1) has the following interpretation. First, consider a straight fluxon tube, say pointing in $\widehat{z}$ (assuming periodic boundary condition). If we translate the entire loop in the $(x, y)$ plane, $T_{x}$ and $T_{y}$ will commute only up to a phase: $T_{y}^{-1} T_{x}^{-1} T_{y} T_{x}=\exp \left(-2 \pi i n_{A} L_{z}\right)$, where $L_{z}$ is the number of layers of the entire system in $\widehat{z}$. Another way to describe this property, without relying on having a finite $L_{z}$, is to consider a closed fluxon loop that links with a dislocation (a line defect in 3D), say with Burgers vector $\widehat{z}$. Translation symmetries will act on the loop projectively. More generally, for a fluxon loop linked with a dislocation with Burgers vector $\mathbf{B}$, we have

$$
T_{j}^{-1} T_{i}^{-1} T_{j} T_{i}=\exp \left(-2 \pi i \epsilon^{i j k} B_{k} n_{A}\right) .
$$

Another consequence, following similar reasoning, is a nonAbelian three-loop braiding [51] for a fluxon loop and two dislocations.

\section{APPENDIX F: SQUARE MODEL NUMERICS TO VERIFY BOUNDARY LUTTINGER THEOREM}

Our square lattice model consists of spinless fermions with nearest neighbor and diagonal hopping, detailed configuration shown in Fig. 3(a). It's modified from $\pi$-flux square hopping with $\epsilon$ variation of vertical hopping, time-reversal breaking imaginary diagonal hopping, and complex diagonal hopping $t$ further breaks remaining rotation (inversion), reflection symmetries to allow a generic polarization. The only symmetry that remains preserved is translation. When put on a cylinder geometry with $x_{2}=0, L$ boundaries and periodic along $x_{1}$ direction, we can calculate boundary charge densities for, e.g., $x_{2}=0$ boundary as [13]

$$
\rho_{b d}=\frac{1}{\Omega} \int_{-\infty}^{x_{0}} d x_{2} \int_{x_{2}-a / 2}^{x_{2}+a / 2} d x^{\prime} \int_{A_{b d}} d x_{1} \rho\left(x_{1}, x^{\prime}\right),
$$

where $\rho\left(x_{1}, x_{2}\right)$ is the charge density including ions (for a neutral system), $x_{0}$ locates deep in the bulk, $a$ is size of unit cell along the $x_{2}$ direction, $A_{b d}$ denotes any segment covering exactly one unit cell on the boundary and $\Omega$ the unit cell area. This amounts to first averaging charge density $\bar{\rho}\left(x_{2}\right)=\frac{1}{\Omega} \int_{x_{2}-a / 2}^{x_{2}+a / 2} d x^{\prime} \int_{A_{b d}} d x_{1} \rho\left(x_{1}, x^{\prime}\right)$ within a window $\left[x_{2}-a / 2, x_{2}+a / 2\right]$ to smoothen any irrelevant periodic oscillations in the bulk $[\bar{\rho}(x)=0$ for $x$ inside the bulk] while retaining the extra charge accumulation [52], then integrating the averaged density. From the field-theoretic point of view, this window function $\bar{\rho}(x)$ use comes naturally from the application of the long-wavelength limit in Eq. (8) to discrete lattices. In continuum, one identifies each unit cell with a single point $\mathbf{x}$ and hence the vector potential $A_{0}(\mathbf{x})$ couples to the average density inside the unit cell $\bar{\rho}(\mathbf{x})$. On the other hand, the lattice-scale oscillation of bare $\rho(\mathbf{x})$ renders it incompatible with the continuum limit in long wavelength. $\rho(\mathbf{x})$ for the boundary charge density, however, matches with $\tilde{\mathbf{P}}$ (see last paragraph).

Similarly, we get boundary $k_{\mathrm{F}}$ as one varies chemical potential. The bulk polarization is calculated by Eq. (B6) For simplicity, we put the positive ions at sites with integral coordinates in units of Bravais lattice vectors, i.e., site $(0,0)$ 

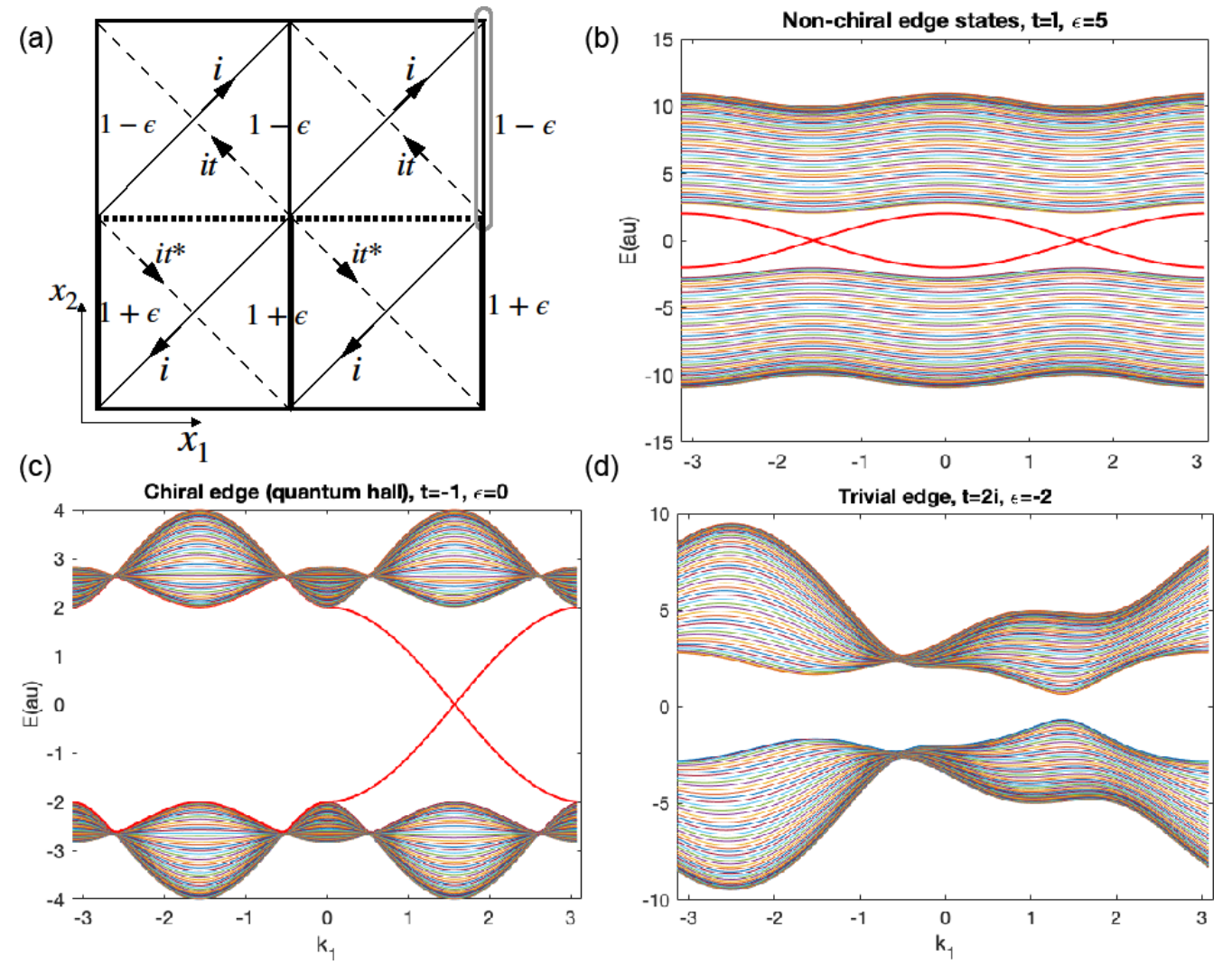

FIG. 3. (a) illustrates the fermion hopping parameters used. The hopping strengths on solid/dotted horizontal bonds are \pm 1 , on normal weight/bold vertical bonds are $1 \mp \epsilon(\epsilon \in \mathbb{R})$ and across solid/dashed diagonal bonds are $i, i t^{(*)}(t \in \mathbb{C})$ (direction denoted by arrows), respectively. The unit cell is doubled along vertical direction. (b)-(d) show typical energy spectrum on a cylinder geometry with boundaries at $x_{2}=0, L$ and periodic in $x_{1}$ direction. The in-gap red lines depict states localized at two edges. (b), (d) satisfy Eq. (18) while (c) has nonvanishing Chern number and gauge-dependent polarization, boundary $k_{\mathrm{F}}$, so boundary Luttinger theorem applies with an appropriate Berry connection integral rule.

and its equivalents by lattice translations. The ions don't contribute to polarization in this way; Eq. (B6) gives the entire polarization then.

As we vary the parameters $t, \epsilon$, the system enters multiple physical regimes with a gapped bulk. For example, the system hosts nonchiral edge states in Fig. 3(b) and when chemical potential stays inside the bulk gap, the edge density $\rho$, Fermi momentum $k_{\mathrm{F}}$, and bulk polarization $P$ always obey Eq. (18). (We note it's important for the bulk to remain insulating with the chemical potential in between the gap.) A relatively trivial scenario in Fig. 3(d) is in the absence of edge states, the edge density equals bulk polarization in line with previous knowledge of polarization. A tricky case is when the model has a nonzero Chern number $C$ shown in Fig. 3(c) and edge density $\rho, k_{\mathrm{F}}$, polarization will change upon a large gauge transform along the orthogonal direction, i.e., gauge dependent. We find that under a fixed gauge, Eq. (18) still holds given appropriate recipe for bulk polarization Eq. (B6) i.e., $2 \pi C$ discontinuity of Wilson loop phase $\theta_{k_{1}}=\int d k_{2}\left\langle u_{\mathbf{k}}\left|i \partial_{k_{2}}\right| u_{\mathbf{k}}\right\rangle$ occurs only at $k_{1}=0$. In all cases, the momenta of a monopole on a torus geometry satisfy $\left(k_{1}, k_{2}\right)=2 \pi\left(-P_{2}, P_{1}\right)$ obtained by the method in Appendix B 1.

Finally, we remark that all calculations above apply also to the intercell part of polarization $\tilde{P}$, when we calculate the boundary charge density as $\rho_{0}=\int_{-\infty}^{n a} d x_{2} \int_{A_{b d}} d x_{1} \rho\left(x_{1}, x_{2}\right) \quad(n \in Z)$ instead of Eq. (F1). The relation between these two reads [16]

$$
\rho_{b d}=\rho_{0}-\frac{1}{a} \int_{n a-a / 2}^{n a+a / 2} d x_{2} \int_{A_{b d}} d x_{1} \rho\left(x_{1}, x_{2}\right) x_{2},
$$

where we take $x_{0}=n a$ in Eq. (F1) and use the neutrality condition. In passing, we remark this rewriting makes explicit the equivalence between the window function method and the charge density as derived in Appendix A. There the charge density in two spatial dimensions reads

$$
\rho\left(\mathbf{x}_{0}\right)=\int_{\Omega_{x_{0}}} d^{2} x \rho(\mathbf{x})-\frac{1}{a} \int_{\Omega_{x_{0}}}\left(\mathbf{x}-\mathbf{x}_{0}\right) \cdot \nabla_{\Omega} \rho(\mathbf{x}),
$$

where $\Omega_{x_{0}}$ is the unit cell at $\mathbf{x}_{0}$ and $\nabla_{\Omega}$ denotes gradient of $\rho$ with respect to its value at the same sublattice in neighboring unit cells. When there's a boundary to vacuum, the boundary charge $\sum_{x_{0}} \rho\left(\mathbf{x}_{0}\right)$ has two parts: the first term integrates to $\rho_{0}$ and the second term integrates to give the bulk electric dipole moment. Hence it agrees with Eq. (F2).

It is now clear that $\rho_{0}$ extracts the excess charge at the boundary. The boundaries we considered preserve complete unit cells in bulk. This extra charge accumulation part depends solely on the inter-unit-cell structure. Hence we use the gauge 
recipe Eq. (B1) to calculate monopole momentum related to $\tilde{P}$, whose $A$ fields reside only on bonds between different unit cells and indeed they agree with the Berry connection integral Eq. (B6) using periodic function $\tilde{u}_{\mathbf{k}}(\mathbf{r})$.
[1] D. Vanderbilt, Berry Phases in Electronic Structure Theory: Electric Polarization, Orbital Magnetization and Topological Insulators (Cambridge University Press, New York, 2018).

[2] R. Resta, Macroscopic polarization in crystalline dielectrics: The geometric phase approach, Rev. Mod. Phys. 66, 899 (1994).

[3] R. Resta and D. Vanderbilt, Theory of polarization: A modern approach, in Physics of Ferroelectrics: A Modern Perspective (Springer, Berlin, 2007), pp. 31-68.

[4] R. M. Martin, Piezoelectricity, Phys. Rev. B 5, 1607 (1972).

[5] C. Kallin and B. I. Halperin, Surface-induced charge disturbances and piezoelectricity in insulating crystals, Phys. Rev. B 29, 2175 (1984).

[6] R. D. King-Smith and D. Vanderbilt, Theory of polarization of crystalline solids, Phys. Rev. B 47, 1651 (1993).

[7] R. Resta and S. Sorella, Many-Body Effects on Polarization and Dynamical Charges in a Partly Covalent Polar Insulator, Phys. Rev. Lett. 74, 4738 (1995).

[8] G. Ortiz, P. Ordejón, R. M. Martin, and G. Chiappe, Quantum phase transitions involving a change in polarization, Phys. Rev. B 54, 13515 (1996).

[9] R. M. Martin and G. Ortiz, Recent developments in the theory of electric polarization in solids, Solid State Commun. 102, 121 (1997), Highlights in Condensed Matter Physics and Materials Science.

[10] R. M. Martin, Comment on calculations of electric polarization in crystals, Phys. Rev. B 9, 1998 (1974).

[11] H. Watanabe and M. Oshikawa, Inequivalent Berry Phases for the Bulk Polarization, Phys. Rev. X 8, 021065 (2018).

[12] R. Resta, Theory of the electric polarization in crystals, Ferroelectrics 136, 51 (1992).

[13] D. Vanderbilt and R. D. King-Smith, Electric polarization as a bulk quantity and its relation to surface charge, Phys. Rev. B 48, 4442 (1993).

[14] G. Ortiz and R. M. Martin, Macroscopic polarization as a geometric quantum phase: Many-body formulation, Phys. Rev. B 49, 14202 (1994).

[15] K. N. Kudin, R. Car, and R. Resta, Berry phase approach to longitudinal dipole moments of infinite chains in electronicstructure methods with local basis sets, J. Chem. Phys. 126, 234101 (2007).

[16] J.-W. Rhim, J. Behrends, and J. H. Bardarson, Bulk-boundary correspondence from the intercellular Zak phase, Phys. Rev. B 95, 035421 (2017).

[17] R. Resta, Quantum-Mechanical Position Operator in Extended Systems, Phys. Rev. Lett. 80, 1800 (1998).

[18] A. A. Aligia and G. Ortiz, Quantum Mechanical Position Operator and Localization in Extended Systems, Phys. Rev. Lett. 82, 2560 (1999).

[19] R. Thorngren and D. V. Else, Gauging Spatial Symmetries and the Classification of Topological Crystalline Phases, Phys. Rev. X 8, 011040 (2018).

[20] I.E. Dzyaloshinskii and G.E. Volovick, Poisson brackets in condensed matter physics, Ann. Phys. 125, 67 (1980).

[21] A.F Andreev and M.Yu Kagan, Hydrodynamcs of a rotating superfluid liquid, Zh. Eksp. Teor. Fiz. 86, 546 (1984).
[22] J. Nissinen and G. E. Volovik, Tetrads in solids: from elasticity theory to topological quantum Hall systems and Weyl fermions, J. Exp. Theor. Phys. 127, 948 (2018).

[23] J. Nissinen and G. E. Volovik, Elasticity tetrads, mixed axialgravitational anomalies, and $(3+1)$-d quantum Hall effect, Phys. Rev. Research 1, 023007 (2019).

[24] Z.-M. Huang, L. Li, J. Zhou, and H.-H. Zhang, Torsional response and Liouville anomaly in Weyl semimetals with dislocations, Phys. Rev. B 99, 155152 (2019).

[25] H Kleinert, Gauge Fields in Condensed Matter (World Scientific, New Jersey, 1989).

[26] N. Manjunath and M. Barkeshli, Crystalline gauge fields and quantized discrete geometric response for Abelian topological phases with lattice symmetry, Phys. Rev. Res. 3, 013040 (2021).

[27] This integrating by part may leave a boundary term which accounts for the change of surface bound charge $\sigma_{i}$ due to spatial variation of polarization through $\sigma_{i}=\mathbf{P} \cdot \widehat{n}$, where $\widehat{n}$ is the normal vector of the surface.

[28] R. Dijkgraaf and E. Witten, Topological gauge theories and group cohomology, Commun. Math. Phys. 129, 393 (1990).

[29] J. Alicea, Monopole quantum numbers in the staggered flux spin liquid, Phys. Rev. B 78, 035126 (2008).

[30] Y. Ran, A. Vishwanath, and D.-H. Lee, A direct transition between a Neel ordered Mott insulator and a $d \_x^{2}-y^{2}$ superconductor on the square lattice, arXiv:0806.2321.

[31] M. Hermele, Y. Ran, P. A. Lee, and X.-G. Wen, Properties of an algebraic spin liquid on the kagome lattice, Phys. Rev. B 77, 224413 (2008).

[32] X.-Y. Song, C. Wang, A. Vishwanath, and Y.-C. He, Unifying description of competing orders in two-dimensional quantum magnets, Nat. Commun. 10, 4254 (2019).

[33] F. Pollmann, A. M. Turner, E. Berg, and M. Oshikawa, Entanglement spectrum of a topological phase in one dimension, Phys. Rev. B 81, 064439 (2010).

[34] X. Chen, Z.-C. Gu, and X.-G. Wen, Classification of gapped symmetric phases in one-dimensional spin systems, Phys. Rev. B 83, 035107 (2011).

[35] N. Schuch, D. Pérez-García, and I. Cirac, Classifying quantum phases using matrix product states and projected entangled pair states, Phys. Rev. B 84, 165139 (2011).

[36] L. Savary and L. Balents, Quantum spin liquids: A review, Rep. Prog. Phys. 80, 016502 (2017).

[37] M. Oshikawa, Topological Approach to Luttinger's Theorem and the Fermi Surface of a Kondo Lattice, Phys. Rev. Lett. 84, 3370 (2000).

[38] E. Lieb, T. Schultz, and D. Mattis, Two soluble models of an antiferromagnetic chain, Ann. Phys. 16, 407 (1961).

[39] M. B. Hastings, Lieb-Schultz-Mattis in higher dimensions, Phys. Rev. B 69, 104431 (2004).

[40] M. Cheng, M. Zaletel, M. Barkeshli, A. Vishwanath, and P. Bonderson, Translational Symmetry and Microscopic Constraints on Symmetry-Enriched Topological Phases: A View from the Surface, Phys. Rev. X 6, 041068 (2016).

[41] M. A. Metlitski and R. Thorngren, Intrinsic and emergent anomalies at deconfined critical points, Phys. Rev. B 98, 085140 (2018). 
[42] Gil Young Cho, C.-T. Hsieh, and S. Ryu, Anomaly manifestation of Lieb-Schultz-Mattis theorem and topological phases, Phys. Rev. B 96, 195105 (2017).

[43] Jeffrey C. Y. Teo, L. Fu, and C. L. Kane, Surface states and topological invariants in three-dimensional topological insulators: Application to $\mathrm{Bi}_{1-x} \mathrm{Sb}_{x}$, Phys. Rev. B 78, 045426 (2008).

[44] S. Coh and D. Vanderbilt, Electric Polarization in a Chern Insulator, Phys. Rev. Lett. 102, 107603 (2009).

[45] G. van Miert and C. Ortix, Dislocation charges reveal twodimensional topological crystalline invariants, Phys. Rev. B 97, 201111(R) (2018).

[46] X.-Y. Song, Y.-C. He, A. Vishwanath, and C. Wang, From Spinon Band Topology to the Symmetry Quantum Numbers of Monopoles in Dirac Spin Liquids, Phys. Rev. X 10, 011033 (2020).
[47] X.-L. Qi, T. L. Hughes, and S.-C. Zhang, Topological field theory of time-reversal invariant insulators, Phys. Rev. B 78, 195424 (2008).

[48] A. M. Essin, J. E. Moore, and D. Vanderbilt, Magnetoelectric Polarizability and Axion Electrodynamics in Crystalline Insulators, Phys. Rev. Lett. 102, 146805 (2009).

[49] E. Witten, Dyons of charge e $\theta / 2 \pi$, Phys. Lett. B 86, 283 (1979).

[50] Y.-M. Lu, Y. Ran, and M. Oshikawa, Filling-enforced constraint on the quantized Hall conductivity on a periodic lattice, Ann. Phys. 413, 168060 (2020).

[51] C. Wang and M. Levin, Braiding Statistics of Loop Excitations in Three Dimensions, Phys. Rev. Lett. 113, 080403 (2014).

[52] K. N. Kudin, R. Car, and R. Resta, Quantization of the dipole moment and of the end charges in push-pull polymers, J. Chem. Phys. 127, 194902 (2007). 